\title{
Electrical Machines Virtual Laboratory: Grid Connection of a Synchronous Generator
}

\author{
Claudia S. Marțis, Horia C. Hedeşiu, Loránd Szabó, Bogdan Tătăranu, Florin Jurcă, Claudiu Oprea \\ Department of Electrical Machines, Technical University of Cluj, Romania
}

\begin{abstract}
Virtual laboratories can become important components of the teaching process, because using them several experiment-oriented problems can be solved easily and also from the distance. Graduates using virtual laboratories will be better trained when they enter the job market. In the paper a laboratory work will be presented having two components: a simulation and an experimental module. Both can be used for the preparation of a real lab and for accessing the real-world experiments. The virtual lab modules can also allow multiple students to remote access the experiments, the multicast data transfer mechanisms and arbitration mechanisms.
\end{abstract}

\section{INTRODUCTION}

"Promotion of the necessary European dimension in higher education, particularly with regards to curricular development, inter-institutional cooperation, mobility schemes and integrated programmes of study, training and research" was set as an objective in the Bologna Declaration.

In the frame of the common education and training activities possibly it could be necessary for students to study at different universities. In this context, student mobility could be seen as a means of acquiring knowledge and skills not available at the home institution, and of complementing and integrating the activities carried out at the home institution.

The need to create equal studying opportunities for all the students implies additional funds that should be provided to cover the higher cost of joint degree programmes. In order to reduce or eliminate these needs, and the overhead incurred when achieving and maintaining a well-equipped laboratory, the new computer technologies and the Internet access can sustain the development of virtual laboratories. As an important component of the teaching process, these could offer experiment-oriented problems to be solved and could give graduates an added value when they enter the European/International job market.

The objective of a virtual laboratory is to introduce students to experimentation, problem solving, data gathering and scientific interpretation. Educators and researchers, crunched between demand for an effective education and budget constrains, can use virtual instrumentation to automate routine tasks, accomplish new objectives, replace outdated and expensive equipment, and/or to demonstrate students the potential of high technology.

For the implementation of the virtual laboratory LabVIEW, a product of National Instruments Inc., will be used. It is a flexible, general-purpose graphical programming tool intended for a broad spectrum of applications. LabVIEW has also controls to access a web page as an interactive interface to an own application, which implements, for example, a data acquisition task on a server. NI-DAQ, also a product of this company, includes a feature called Remote Device Access (RDA), which allows remote, network access to data acquisition systems using the company's wide-spread hardware.

The Electrical Machines Virtual Laboratory integrates computers and industrial hardware expanding the range of instrumentation and control to virtually unlimited capabilities, offering for the students a pre-practice, a replacement or a post-analysis of the real laboratories.

The methodology used in the Electrical Machines Virtual Laboratory is structured in a same manner as in the case of the real lab. It begins with an introduction containing a short discussion on the laboratory exercise to be performed (including details on data collection and data analysis), procedures (instructions on how to conduct the laboratory work), respectively a step-by-step guide through the virtual lab, analysis, report writing requirements and references.

\section{THE VIRTUAL LAB}

Connecting a generator to an ac power network is the synchronisation of the generator to the grid. Before this procedure the generator has to be accelerated to the correct speed and the rotor must be magnetized.

The current $I$ resulting at the moment of synchronization depends on how close the induced voltage $\left(E_{q}\right)$ and the terminal voltage $(V)$ are each to other. To avoid large circulating currents the phasor values of $E_{q}$ and $V$ should be equal. This requires four conditions: same magnitude, phase and frequency of $E_{q}$ and $V$, respectively same phase order of both three-phase systems.

\section{A. The simulation module}

In the first part of the virtual laboratory work concerning the grid connection of a synchronous generator the simulation of the voltage waveforms of the two components of the system (the generator and the power grid) will be done.

By varying all the parameters (amplitude, frequency, phase and phase order) of the two voltage systems different cases can be analysed from the worst situation, when neither of the four conditions are fulfilled, to the ideal case, when the connection to the grid could be done.

In Fig. 1 the block diagram of the simulation program is given. The amplitudes $\left(A_{n}, A_{g}\right)$ and the frequencies $\left(f_{n}, f_{g}\right)$ of the two sources corresponding to the network, respectively to the synchronous generator can be set independently. The two waveforms are synchronised by imposing in both cases the same step number $i$. 


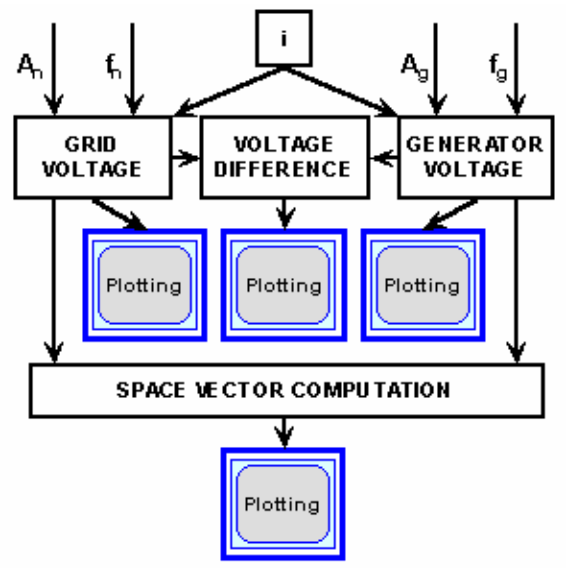

Fig. 1. Block diagram of the simulation program.

The simulation program is practically a LabVIEW virtual instrument. On its front panel (see Fig. 2) all the four parameters of the two voltage waveforms can be modified easily. The front panel also contains four waveform charts for plotting the two three-phase voltage

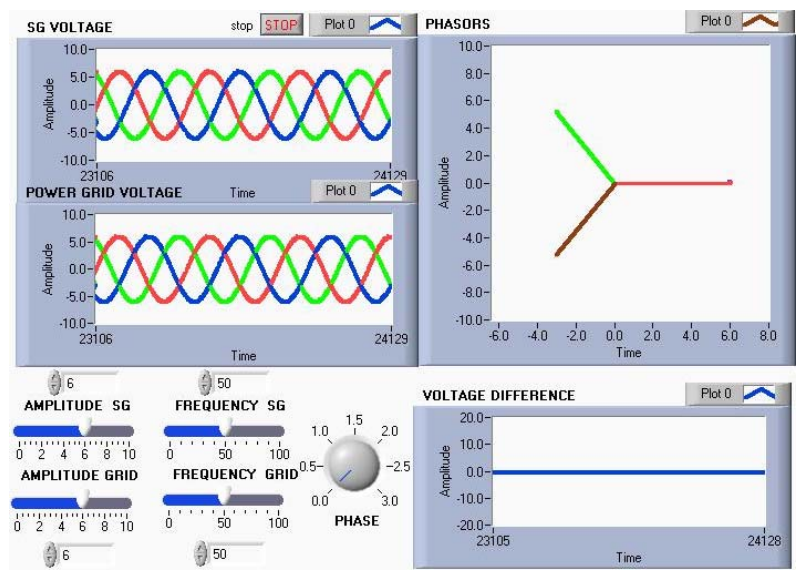

a) case $\mathrm{A}$

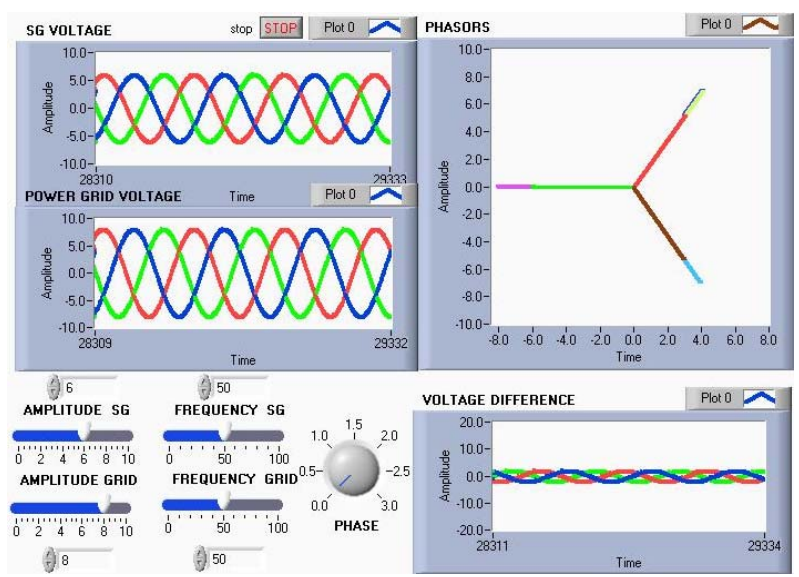

c) case $\mathrm{C}$ systems, the resulting space vectors, and the voltage differences.

All the possible situations were studied using this virtual instrument. Here only the results of the four most significant cases (see Table I.) are given. The results, as obtained on the front panel of the virtual instrument, are given in Fig 2.

TABLE I.

THE FOUR CASES IN STUDY

\begin{tabular}{|c|c|c|c|c|c|}
\hline \multirow[t]{2}{*}{ Case } & \multicolumn{5}{|c|}{ Coupling conditions fulfilment } \\
\hline & 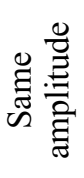 & 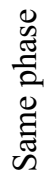 & 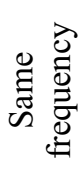 & 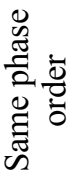 & 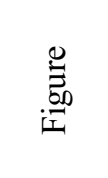 \\
\hline $\mathbf{A}$ & $\mathrm{X}$ & $\mathrm{X}$ & $\mathrm{X}$ & $\mathrm{X}$ & Fig. 2a \\
\hline $\mathbf{B}$ & $\mathrm{X}$ & $\mathrm{X}$ & $\mathrm{X}$ & - & Fig. $2 b$ \\
\hline $\mathbf{C}$ & - & $\mathrm{X}$ & $\mathrm{X}$ & $\bar{X}$ & Fig. 2c \\
\hline D & - & - & - & - & Fig. $2 \mathrm{~d}$ \\
\hline
\end{tabular}

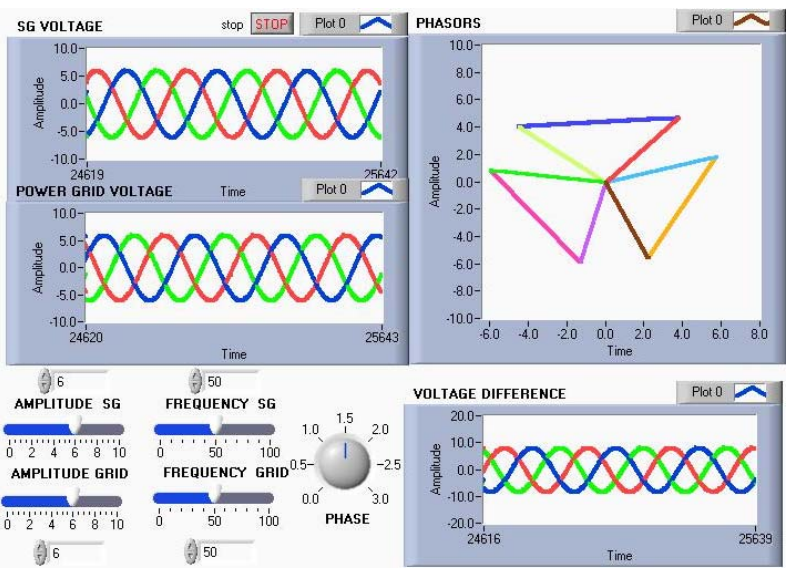

b) case $\mathrm{B}$

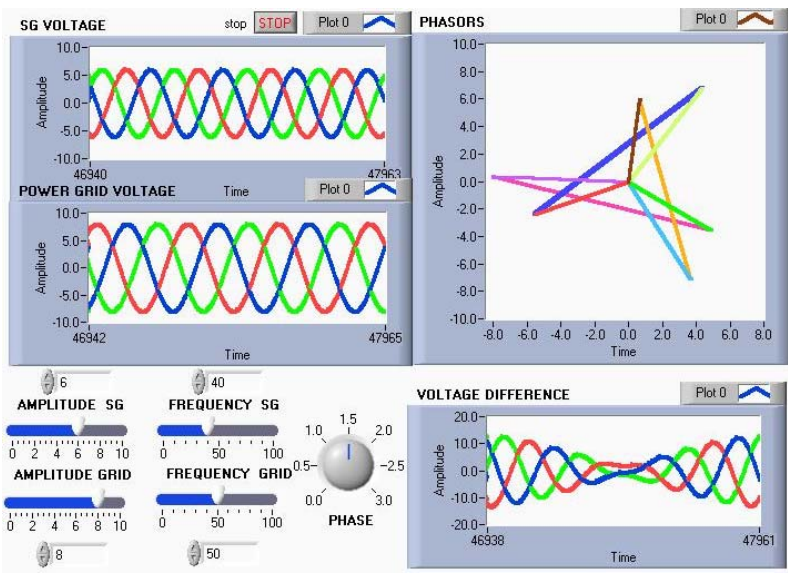

d) case D

Fig. 2. The results of simulation on the front panel of the virtual instrument.

The simulation module of the virtual lab in discussion gives students the possibility to investigate all the possible combinations of the four quantities in study before coupling a generator to the grid. They will be able to see and understand the effects of the difference between the amplitudes, the frequencies, the phases or of the phase order of the two 3-phase voltage systems.

After this study the students will be able to connect in practice a synchronous generator to the grid. 


\section{B. The experimental test-bench}

The scheme of the conventional experimental set-up used to study the synchronization of a synchronous generator with the three-phase grid is given in Fig. 3a. The frequencies of the two voltages are measured using two frequency meters $\left(\mathrm{Hz}_{1}\right.$ and $\left.\mathrm{Hz}_{2}\right)$, the amplitudes of the voltages using two voltmeters $\left(V_{l}\right.$ and $\left.V_{2}\right)$, respectively the phase order is checked by a synchronouscope $(S)$.

The scheme of a more advanced experimental set-up using data acquisition system is given in Fig. 3b. As it can be seen, the number of the apparatus is significantly reduced.

The conventional measuring unit and the synchronouscope are replaced by three voltage transducers. These measure the voltage differences between each phase of the generator and the corresponding phase of the grid. The voltage transducers are connected to the data acquisition board via a CSXI signal conditioning unit. The entire measuring system is controlled from the LabVIEW environment.

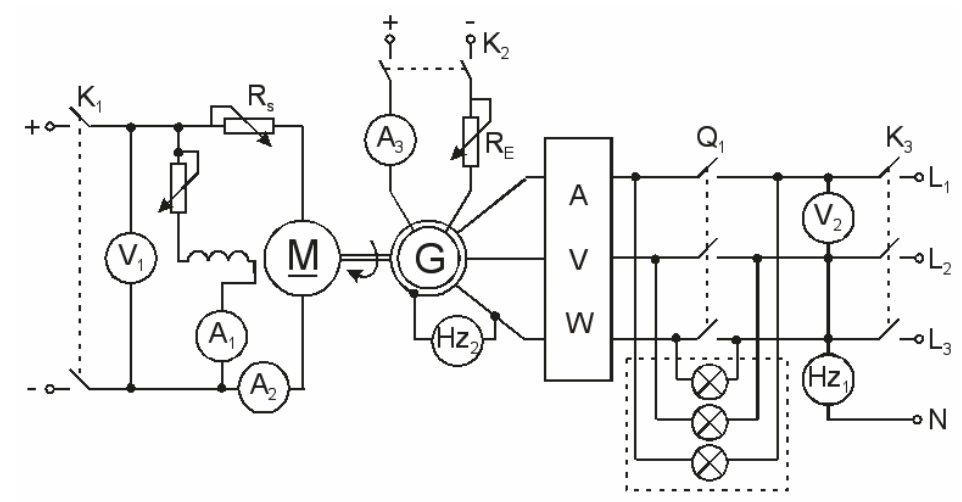

a) with conventional measuring system

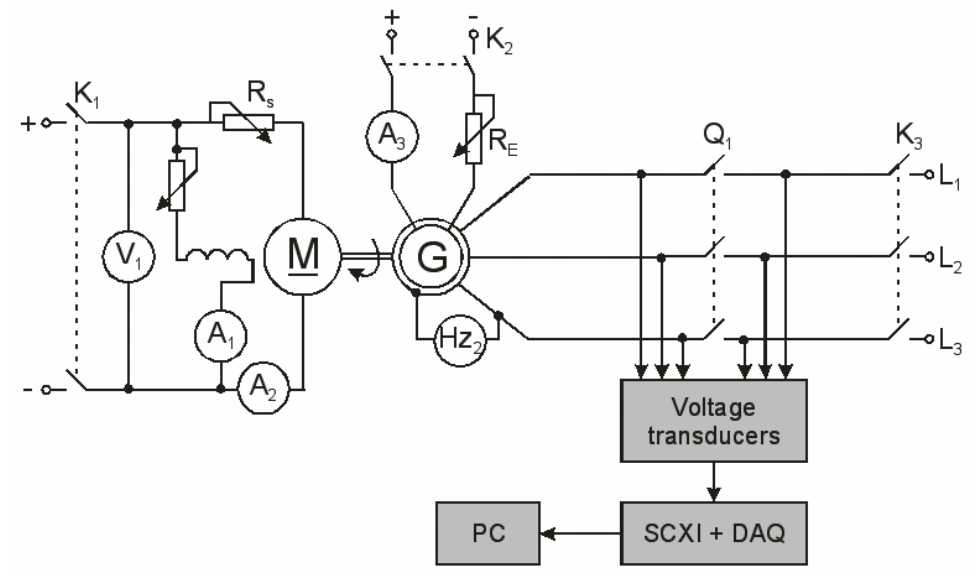

b) with data acquisition system

Fig. 3. The scheme of the experimental set-up.

The results of the Fast Fourier Transformation (FFT) of the measured voltage differences give valuable information on their magnitude and frequency.

The variance of the measured voltage differences can give information the users on the phase orders of the two voltage systems in study.

As it is known from statistics the variance is a measure of how spread out a distribution is. It is computed as the average squared deviation of each measured value from its mean:

$$
S^{2}=\frac{\sum(X-M)^{2}}{N-1}
$$

where $N$ is the sample number of $X$, respectively $M$ is the mean value of the sample.

By means of simulation the effect of the phase orders of the two three-phased voltage systems on the variance of the voltage differences were studied.

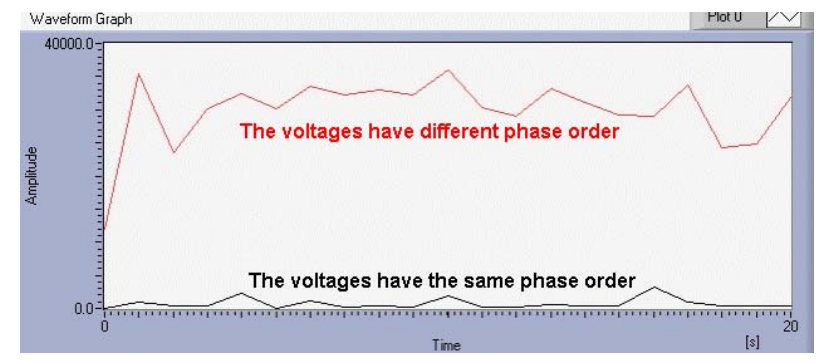

Fig. 4. The variance of the voltage difference.

As it can be seen in Fig. 4 the variance of the voltage difference in the case of the same phase order is much smaller than in the case when these are different.

All this experiences were taken into account when the virtual instrument of the experimental test bench was built up. This unit beside the data acquisition also processes the measured data (by means of FFT and of the variance's computation), and plots the results. 


\section{THE LABVIEW-BASED EXPERIMENTS}

A picture of the developed experimental set-up having the both measuring systems given in Fig. 3 can be seen in Fig. 5.

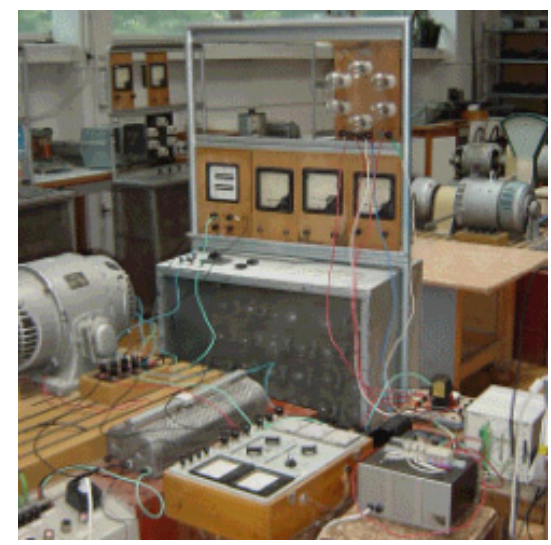

Fig. 5. The laboratory set-up

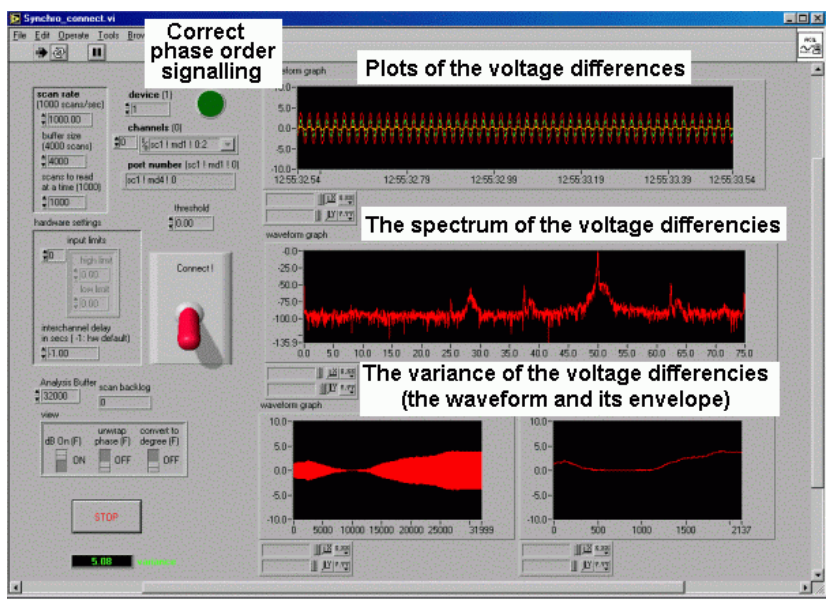

Fig. 6. The front panel and the block diagram of the virtual instrument.

Next results of the laboratory experiences done with the above presented LabVIEW virtual instrument are given.

First the worst situation was studied (case D from Table I), when neither one of the four conditions were fulfilled. In Fig. 7 four plots are given: the variation of the three voltage differences versus time, the power spectrum of one of these differences, the variation of its variance computed upon equation (1), respectively the envelope of the variance's shape.

As it can be seen in Fig. 7 in this case the voltages have different phase order, frequency and amplitude. In the spectrum of the voltage difference (Fig. 7b) the two components due to the $50 \mathrm{~Hz}$ and $47.5 \mathrm{~Hz}$ frequencies of the grid, respectively of the generator can be distinguished. The other components are parasites coming from the grid.

The great variations seen in the shape of the variance's envelope (Fig. 7d) indicate that the generator cannot be coupled in these conditions to the grid.

Next results from studying another case will be presented: when only the phase order similarity is not fulfilled (case B from Table I). The different phase sequence can be observed easily in Fig. 8a, where the
The synchronisation with the grid of a $4.5 \mathrm{~kW}, 4$ poles synchronous generator was experimented.

A dc motor was used to rotate the synchronous generator at different speeds in order to vary its frequency. Changing the excitation current of the synchronous generator the amplitude of the generator's voltage was changed.

These modifications were observed on both measurement schemes: the conventional one and the LabVIEW-based one, respectively.

The front panel of the LabVIEW program is given in Fig. 6. On it the waveform of the voltage differences and the voltage difference spectrum can be visualised. Also the plots versus time of the voltage difference's variance and its envelope are plotted here (see Fig. 8).

A lamp placed on the front panel is used to give a signal when the variance analysis enables the connection to the grid. The connection can be performed by pressing a button from the front panel. The generator may be coupled also automatically.

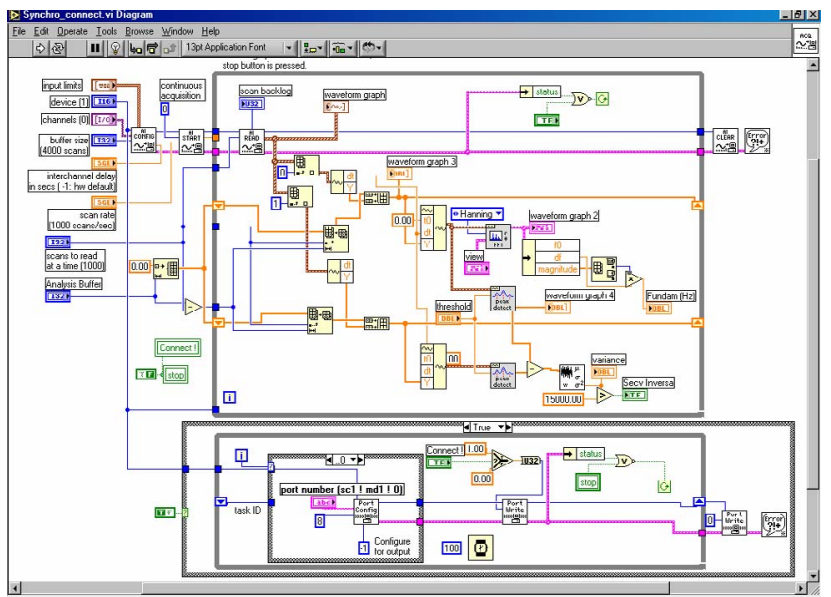

three voltage differences are plotted versus time. The power spectrum of the voltage difference shows clearly that both voltages have the same frequency $(50 \mathrm{~Hz})$.

Similarly to the above presented case, also now the envelope of the voltage difference's variance has great ripple, which shows that not all the conditions are fulfilled for connecting the synchronous generator in study to the three-phase power grid.

In Fig. 9 the results when all the requirements for coupling the generator to the grid (case A from Table I) are shown. The plots of the voltage differences display the perfect equality of the two voltages, both in amplitude and phase.

The spectrum of the voltage having a single emphasized component at $50 \mathrm{~Hz}$ also indicates the equality of the two frequencies. Also the variance of the voltage differences in Fig. 9c shows that the generator can be coupled in these circumstances.

Hence the generator was connected to the grid. The short transient period followed after the coupling can be clearly observed in Fig 9c. Only during this period has the envelope of the voltage difference's variance great values. 


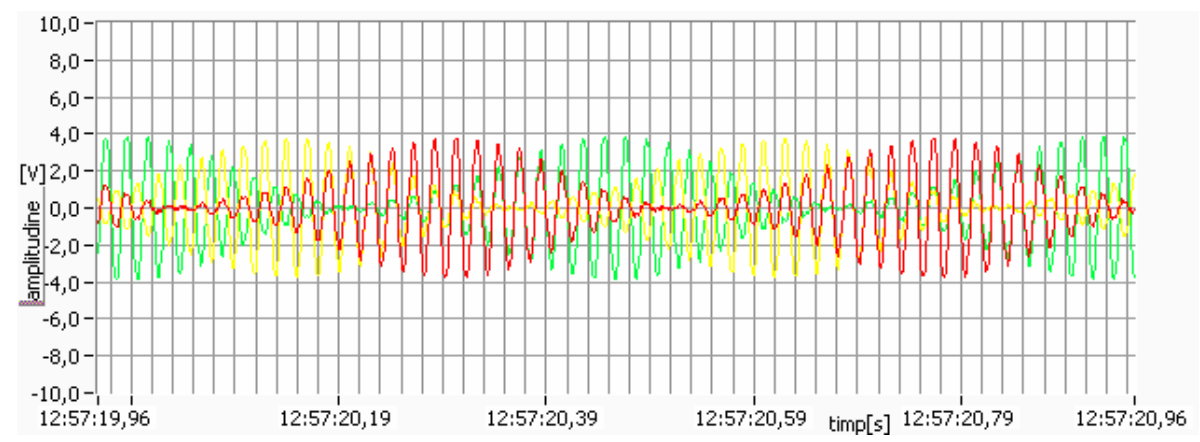

a) The plots of the voltage differences

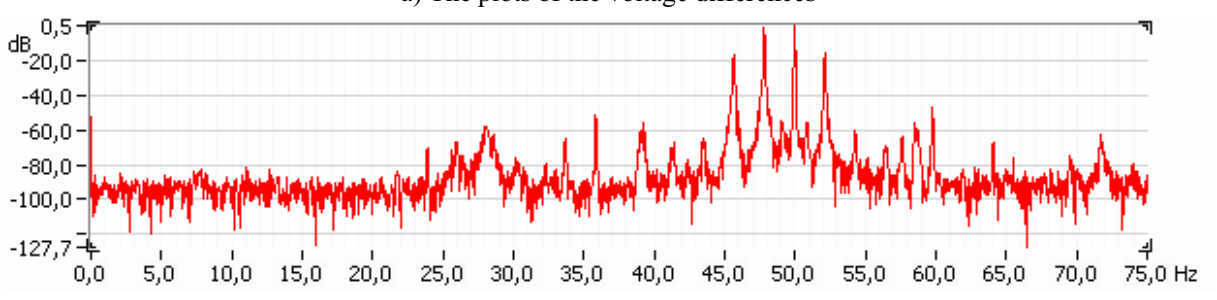

b) The spectrum of a voltage difference

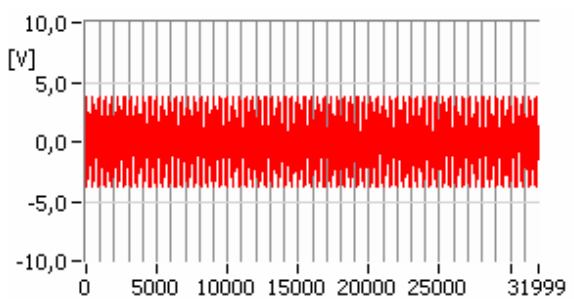

c) The variance of a voltage difference

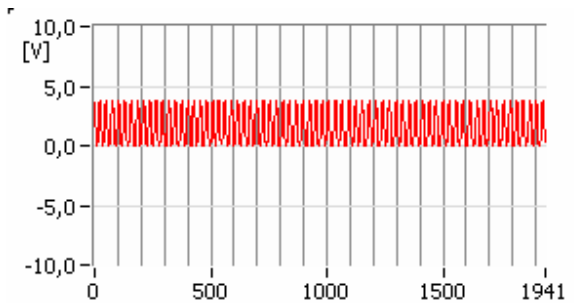

d) The envelope of the variance's shape

Fig. 7. Waveforms taken from the virtual instrument for case D.

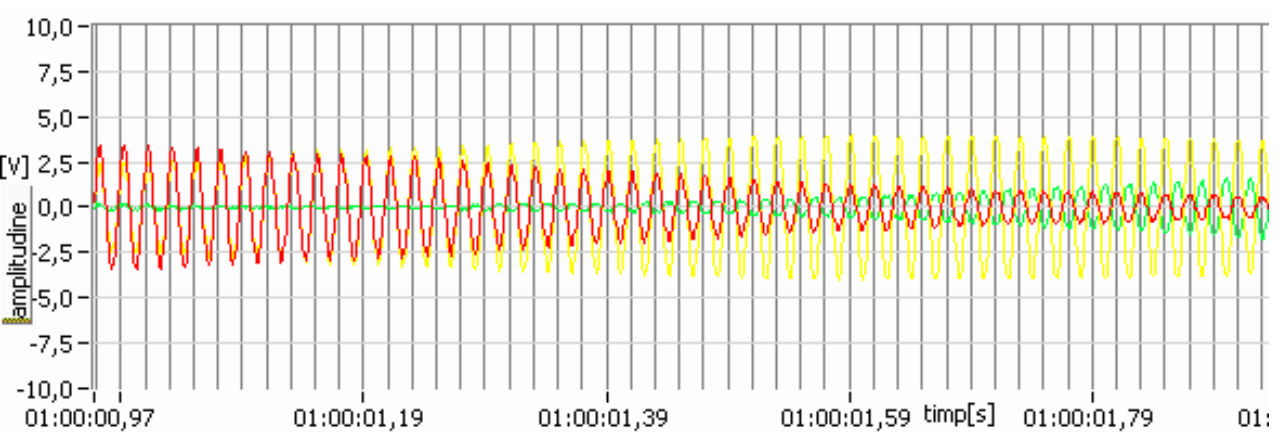

a) The plots of the voltage differences

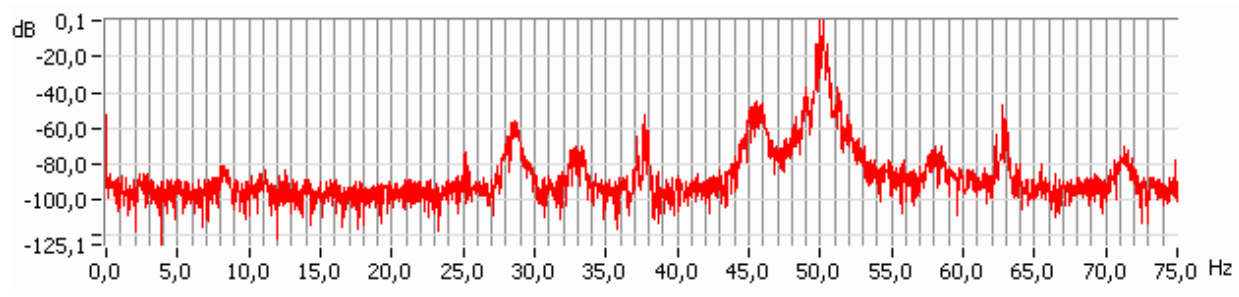

b) The spectrum of a voltage difference
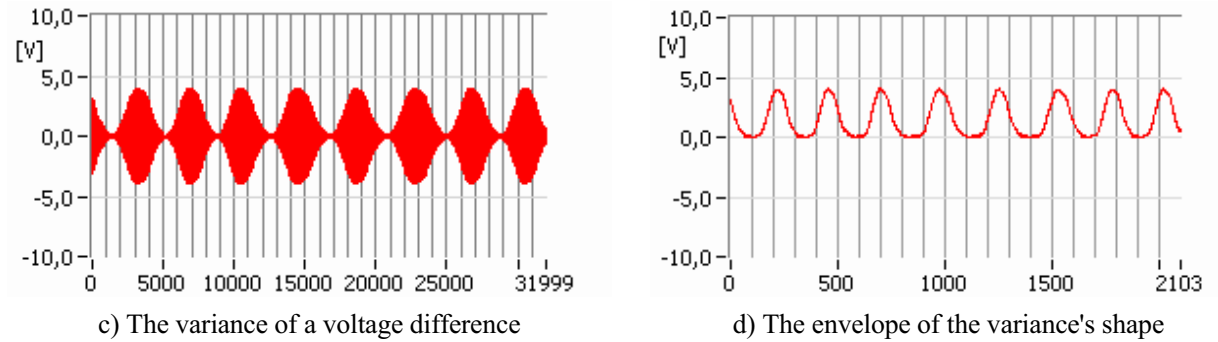

d) The envelope of the variance's shape

Fig. 8. Waveforms taken from the virtual instrument for case B. 


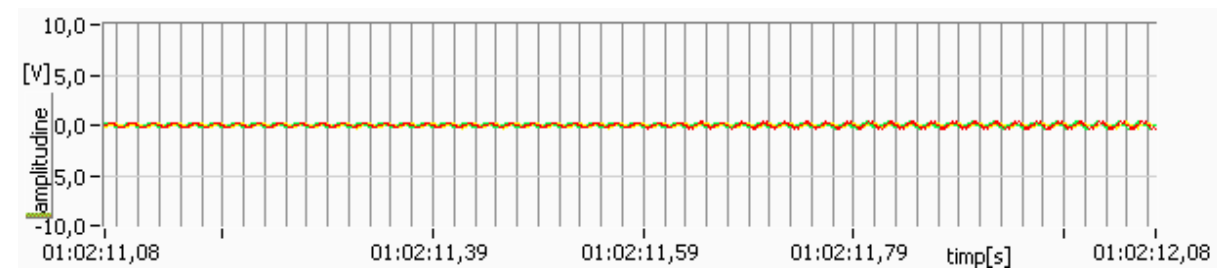

a) The plots of the voltage differences

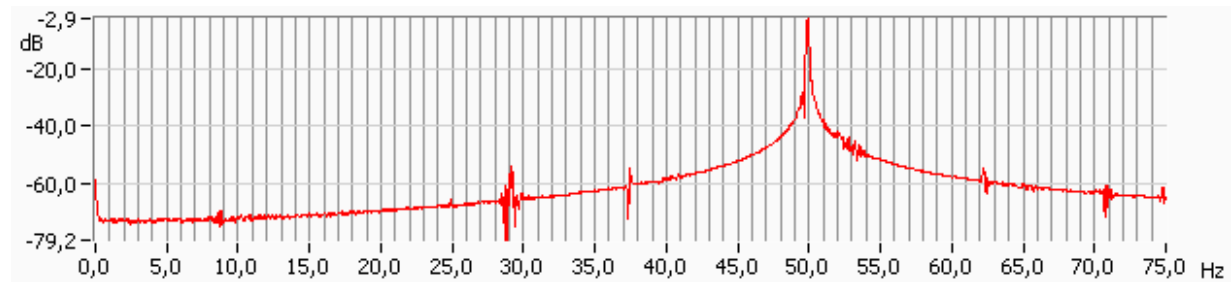

b) The spectrum of a voltage difference

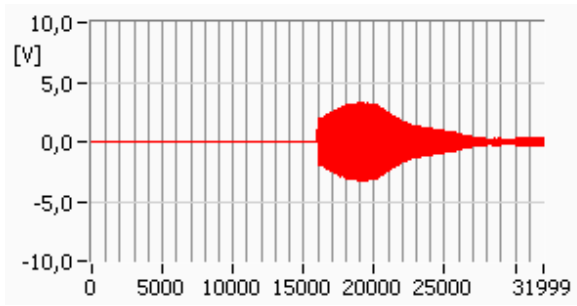

c) The variance of a voltage difference

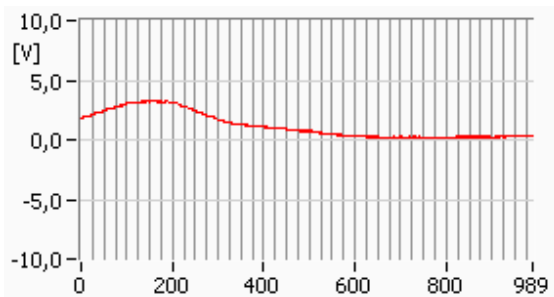

d) The envelope of the variance's shape

Fig. 9. Waveforms taken from the virtual instrument for case A.

Using this very practical virtual instrument coupled to a laboratory set-up a lot of experiences can be gathered, regarding how the different amplitudes, phases and phase orders influence the shape of the voltage differences and implicitly its power spectrum.

It was also observed, that the difference of the voltage amplitudes has an effect on the power spectrum of the voltage difference. In this case more harmonic components can be observed beside the main one of $50 \mathrm{~Hz}$.

It was proved that the envelope of the variance signal of the voltage differences can be used as a sign of the four required condition's fulfilment.

After some practice with this virtual instrument co-ordinated set-up the students will surely better understand the physical phenomena taken place, respectively why the imposed requirements must be fulfilled.

\section{CONCLUSIONS}

Internet offers new non-traditional possibilities how to improve and complete the classical educational methods. Hence the presented laboratory work will be extended in a manner to be accessed also from distance using the Internet.

The electrical engineering students can use the Electrical Machines Virtual Laboratory in order to prepare themselves for the real laboratory exercises.

The simulation module of the presented virtual laboratory work can be realized as a stand-alone component, which gives the possibility of study the grid connection of a synchronous generator to the three-phase ac network.
Meanwhile the students will have access to files containing information on the basic principles of the measuring method, the basic parameters of the system in study and also to the files where the students can change the parameters and watch how the system's behaviour is influenced by this.

The virtual laboratory module will contain assessment, too. Each of them will contain several different questions bearing with the discussed topics: electrical machine's principles, parameters that may be used in simulations, or the basic properties of the measuring method. For each question a single correct answer have to be selected from a set of possible answers.

Many kinds of multimedia, communication and information sharing methods can be used in modern electrical engineering teaching. The intelligent experimental set-up presented in the paper could provide a kind of access to real experiments of multiple remote users.

\section{REFERENCES}

[1] Gustavsson, I., "A Remote Access Laboratory for Electrical Circuits Experiments," International Journal on Engineering Education, vol. 19 (special issue on Distance, Controlled Laboratories and Learning Systems), no. 3, pp. 409-419, 2003.

[2] Grätz, P., Šedivá, S., "Virtual Laboratory of Sensors and Measurement of Non-electrical Quantities," CEEPUS Cz-103 International Summer School ACTA '2003.

[3] Saliah, H.H., Abecassis, A., Nurse, E., "Design of a Generic, Interactive, Virtual and Remote Electrical Engineering Laboratory," Proceedings of the $29^{\text {th }}$ ASEE/IEEE Frontiers in Education, San Juan (Puerto Rico), pp. 255-262, 1999.

[4] "LabVIEW User Manual," National Instruments, Austin, 2000

[5] Gillet D. et al., "Remote Manipulation with LabVIEW for Educational Purposes," in: Internet Applications in LabVIEW, Prentice Hall PTR, Upper Saddle River, 2000. 